\title{
Review: psychosocial treatments can play an important part in the comprehensive management of patients with schizophrenia
}

\author{
Mojtabai R, Nicholson RA, Carpenter BN. Role of psychosocial treatments in management of schizophrenia: a meta-analytic review \\ of controlled outcome studies. Schizophr Bull 1998;24:569-87.
}

\section{Question}

In patients with schizophrenia, what are the effects of adding psychosocial treatments to standard medical treatments?

\section{Data sources}

Studies were identified by searching Medline (1966-94) and PsycLIT (1974-94), handsearching journals that had published most of the identified studies, and by reviewing the references of previous reviews.

\section{Study selection}

Studies were selected if there was a comparison of $\geqslant 2$ groups of patients with a diagnosis of schizophrenia and at least 1 group received a form of psychosocial treatment. Psychosocial interventions that were of little clinical relevance were excluded (eg, training patients on psychometric tests).

\section{Data extraction}

Data were extracted on study design and location, patient and treatment characteristics, and outcome measures. Outcomes in studies were translated into a standardised estimate of effect size using Cohen's $d$.

\section{Main results}

106 studies were included in the preliminary analysis. Effect sizes for 71 studies which compared combination treatment with standard treatment ranged from -0.48 to 2.23 . The weighted least squares average of the effect sizes was 0.39 (95\% CI 0.32 to
0.44). An effect size of 0.39 implies that a typical patient in the experimental group was better off than $65 \%$ of control patients. 14 studies reported the effects of treatment on relapse. On average, relapse frequencies were $20 \%$ lower for patients who received psychosocial treatment in addition to standard treatment compared with standard treatment alone. Potential moderator variables, which were derived during the data extraction, were examined for their influence on the outcome of studies. Univariate analysis identified significant effects for modality of treatment (individual and family therapy produced some of the largest effect sizes, whereas group therapy produced the smallest effect sizes), and maintenance of treatment effects at follow up. Multiple regression analysis identified a small number of variables which accounted for $62 \%$ of the variance in effect sizes between studies. Firstly, smaller effect sizes were found for studies which used measures of disorganised behaviour and employment. Secondly, larger effect sizes were found in studies with more chronic patients, for studies using objective diagnostic criteria, and for studies from non-Western countries.

\section{Conclusion}

Psychosocial treatments augment and complement the effects of medication in schizophrenia, especially in more chronic stages of illness.

\section{Source of funding: no external funding.}

For correspondence: $\operatorname{Dr} R$ Mojtabai, Division of Epidemiology and Community Psychiatry, Department of Psychiatry, Columbia University, $601 \mathrm{~W}$ 168th Street, Suite \#32, New York, NY 10032, USA.Fax +12127959768.Email rm322@columbia.edu.

\section{Commentary}

The introduction of psychosocial interventions into routine care has been slow. Tarrier et al suggest that this has been because of various barriers, including lack of partnership between researcher and clinician, absence of appropriate knowledge and clinical skills, and organisational characteristics that constrain new developments. ${ }^{1}$

The review by Mojtabai $e t$ al sets out to examine the evidence for the role of psychosocial treatments in the management of schizophrenia. This is an ambitious meta-analytic review, which encompasses a range of psychosocial interventions including behavioural interventions for social skills, family interventions for relapse, cognitive programmes for the rehabilitation of neuropsychological deficits, and psychodynamic psychotherapies.
The results of their analysis show effect sizes ranging from 0.27 for psychodynamic psychotherapies to 0.56 for family interventions. Although this difference is not statistically significant, it suggests an advantage for family therapy focused on relapse prevention. In terms of outcomes, the largest effect sizes were found for negative symptoms $(0.51)$, thought disorder (0.59), and relapse (0.46).

Although this review achieves its aim in promoting the role of psychosocial interventions, there is a key limitation. Within this meta-analytic review it is not possible to distinguish between the specific effects of different types of interventions. This is an important consideration for service planning and commissioning because specific psychosocial interventions are tailored to specific problems associated with schizophrenia. In this respect, there remains a need for further meta-analyes such as Mari and Streiner's review which evaluated the effectiveness of family interventions for relapse. ${ }^{2}$ These kinds of reviews are critical in making the case for the provision and dissemination of particular types of interventions.

Andrew Gumley, BA, MAppSci Anxiety and Stress Research Centre, University of Stirling Stirling, UK

1 Tarrier N, Haddock G, Barrowclough C. Training and dissemination: research to practice in innovative psychosocial treatments for schizophrenia. In: Wykes T, Tarrier N, Lewis S, editors. Outcome and innovation in the psychological management of schizophrenia. New York, NY: John Wiley \& Sons, 1998.

2 Pharoah FM, Mari JJ, Streiner DL. Family interventions for schizophrenia. Cochrane Review, latest version 24 February 1999. In: Cochrane Library. Oxford: Update Software. 